Published in final edited form as:

Curr Opin Cardiol. 2016 May ; 31(3): 235-241. doi:10.1097/HCO.0000000000000274.

\title{
Genetics of congenital heart disease
}

\author{
Jonathan J. Edwards ${ }^{a, b}$ and Bruce D. Gelb ${ }^{a, b}$ \\ aThe Mindich Child Health and Development Institute, Icahn School of Medicine at Mount Sinai, \\ New York City, New York, USA \\ ${ }^{b}$ Department of Pediatrics, Icahn School of Medicine at Mount Sinai, New York City, New York, \\ USA
}

\begin{abstract}
Purpose of review-The goal of this review is to highlight recent discoveries in the field of genetics as it relates to congenital heart disease (CHD). Recent advancements in next generation sequencing technology and tools to interpret this growing body of data have allowed us to refine our understanding of the molecular mechanisms that result in CHD.
\end{abstract}

Recent findings-From multiple different study designs, the genetic lesions that cause CHD are increasingly being elucidated. Of the more novel findings, a forward genetic screen in mice has implicated recessive inheritance and the ciliome broadly in CHD pathogenesis. The developmental delays frequently observed in patients with CHD appear to result from mutations affecting genes that overlap heart and brain developmental regulation. A meta-analysis has provided clarity, discriminating pathologic from incidental copy number variations and defining a critical region or gene.

Summary-Recent technological advances have rapidly expanded our understanding of CHD genetics, and support the applicability to the clinical domain in both sporadic and inherited disease. Though significant gaps remain, genetic lesions remain the primary explanation for CHD pathogenesis, although the precise mechanism is likely multifactorial.

\section{Keywords}

congenital heart disease; genetics; next generation sequencing; oligogenic

\section{INTRODUCTION}

The contribution of genetic variants to the pathogenesis of congenital heart disease (CHD) has been long suspected and, more recently, well established. The accepted model for the roles of genetic variation causing CHD has evolved over time, with the pendulum swinging between complex, summative polygenic models and simplistic, high-impact monogenic ones. The identification of new CHD genes, alleles, and pathways has benefited

Correspondence to Bruce D. Gelb, MD, The Mindich Child Health and Development Institute, Icahn School of Medicine at Mount Sinai, One Gustave L. Levy Place, Box 1040, New York City, NY 10029, USA., Tel: +1 212824 8938; fax: +1 212 241 3310; bruce.gelb@mssm.edu.

Conflicts of interest

There are no conflicts of interest. 
tremendously from the growth of high-throughput genomic technologies, particularly massively parallel DNA sequencing, and evolving methods to interpret these results. Still, significant gaps remain. We propose that this new era of discovery genetics will fill the gaps with evidence of an oligogenic or oligo-factor model wherein discrete genetic and possibly environmental factors operate in concert to perturb normal heart development. This review will discuss recent literature describing attempts to unravel the genetic mechanisms of CHD pathogenesis and address important knowledge gaps identified in these studies.

\section{INHERITED CONGENITAL HEART DISEASE}

In principle, inherited CHD variants cannot be autosomal dominant and eliminate reproductive fitness. Otherwise, their molecular pathogenesis should not inherently differ from sporadic CHD. However, dichotomizing inherited from sporadic cases allows investigators to implicate novel genetic variants and derive genotype-phenotype correlations from pedigree analysis. Complicating matters, though, one must consider confounders including genetic or environmental factors - and low-penetrant variants that can limit this strategy.

\section{KEY POINTS}

- For much of CHD, multiple pathologic factors - which are primarily genetic but also include environmental exposures - must interact to establish pathogenesis.

- Recessive inheritance may play a substantive role in CHD, particularly in cases of intrauterine fetal demise.

- The pathogenicity of CNVs in CHD can be defined by the gene or region that is recurrently impacted in overlapping lesions, and these can be highlighted as candidate genes.

- The shared genetic regulation of heart and brain development is a leading contributor to the observed clinical overlap of CHD with developmental delay.

Three recent studies took similar approaches to identify novel variants of known or candidate CHD-associated genes and simultaneously illustrated the clinical utility of next generation sequencing. In one study, the coding regions of GATA4, NKX2-5, ZIC3, and $E L N$ (for supravalvar aortic stenosis) were sequenced and microarray analysis was performed to assess copy number variation (CNV) at the 22q11 region, BMP4, CRELD1, $N K X 2-5$, and $T B X 5$ for 154 families with at least two individuals affected with CHD [1"]. Using this approach, the authors identified a likely causative lesion in approximately $10 \%$ of the families, with $N K X 2-5$ and $Z I C 3$ being the most commonly altered genes. Additionally, in at least one pedigree, the causative variant (deletion of $N K X 2-5$ ) was observed as de novo in the parental generation but inherited by the proband.

For two additional studies, the coding and splice site regions of 57 previously implicated syndromic and nonsyndromic CHD genes compiled from CHD Wiki (http:// www.esat.kuleuven.be/ bioiuser/chdwiki) were sequenced and variants were filtered in silico [2,3"]. Jia and colleagues [3"] identified causal variants affecting NOTCH1, TBX5, and 
MYH6 in six of the 13 families exhibiting nonsyndromic septal defects. Blue and colleagues [2] identified five causal variants in 16 families altering TBX5, NOTCH1, TFAP2B, and $E L N$ in nonsyndromic familial CHD. Additional gene variants identified in these studies were predicted to be damaging by in silico analysis but failed to segregate with disease, and their functional impact was not delineated. These additional variants were clearly insufficient to cause CHD by themselves, but a contribution to CHD pathogenesis or influence on CHD phenotype could not be excluded.

Conclusively outlining the molecular mechanisms that determine genotype-phenotype correlations is often not straightforward, even in smaller studies that focus on a single CHD phenotype and candidate gene [4]. Bicuspid aortic valve (BAV) is the most common congenital heart malformation but is often clinically silent until late adulthood. Variants affecting the only two genes strongly linked to BAV, NOTCH1 and GATA5, account for a small fraction of cases [5]. A nonsense mutation affecting $N K X 2-5$ (p. K192X) was identified in a patient with inherited BAV and demonstrated in vitro to negate synergistic transcriptional activation with GATA5. Sequencing of available family members identified the mutation in the proband's sister and father, both of whom had BAV as part of a more complex phenotype, including atrial septal defect, paroxysmal atrial fibrillation, and atrioventricular conduction delay.

In a less biased approach, another study used a cost-cognizant method of combinatorial pooling and targeted multigene sequencing of 97 genes associated with heart valve development in 78 unrelated patients with a mix of sporadic and inherited BAV with and without coarctation of the aorta [6]. After filtering in silico and confirmation with Sanger sequencing, 31 putatively pathogenic variants from 28 genes in 16 patients were identified. Only two of these variants were de novo (affecting APC and GATA5), and both were found in the same patient with a family history of aortic coarctation. Pathway analysis of the 28 genes was performed using the Database for Annotation, Visualization and Integrated Discovery, revealing overrepresentation of WNT signaling pathway genes (WNT4, PPP3CA, NFATC1, APC, AXIN1, and AXIN2). Eleven of the remaining 15 patients had no family history of CHD, suggesting that these inherited variants were not sufficient to impact aortic valve development alone but interacted with other genetic or environmental factors in the pathogenesis of BAV.

\section{SPORADIC CONGENITAL HEART DISEASE}

Logically, one would predict that sporadic cases of CHD would not tend to result from inherited variants, aside from rare autosomal inheritance but, instead, would arise from de novo mutations or strong environmental pressure. The work of Zaidi and colleagues [7"] has been reviewed previously in this journal. The interested reader is referred to [8]. Briefly, in a quest to characterize the role of de novo point mutations in sporadic CHD, whole exome sequencing (WES) was performed on 362 sporadic severe CHD trios and 264 control trios, revealing an increased burden of mutations affecting epigenetic processes - namely histone modification. Based on these findings, the authors of that study estimate that de novo mutations in about 400 genes can explain approximately $10 \%$ of sporadic CHD. 
Recessively inherited variants may be an under-recognized contributor to the remaining cases of sporadic CHD [10]. Li and colleagues [9"'] recently performed an N-ethyl-Nnitrosourea (ENU) muta-genesis forward genetic screen of inbred mice that recovered 218 mutant lines exhibiting a wide range of CHD identified by fetal echocardiography (Fig. 1). Subsequent WES identified 91 autosomal recessive mutations across 61 genes. Because genetic observations from mouse models may not ultimately prove relevant for human disease, one cannot yet be assured that these novel genes contribute to CHD in patients. Nonetheless, there are multiple exciting findings worth highlighting. Many of the 61 genes with mutations were previously associated with embryonic lethality in mouse knockouts, but not heart defects, as postnatal cardiac phenotyping could not be performed in those studies. Although both an excess of left-right patterning defects and mutations affecting ciliome genes (34 of 61) were observed, the two were not completely overlapping, implicating the ciliome in CHD pathogenesis even in the absence of heterotaxy. Furthermore, this suggests that situs defects may be overrepresented in recessively inherited variants or in cases of fetal demise. Well-established cardiac developmental pathways also harbored recessive mutations, implicating this inheritance pattern for genes involved in Shh, Wnt/planar cell polarity, $T g f \beta / B m p$, and calcium dynamics. Collectively, these findings are potentially most relevant for the poorly studied forms of CHD that cause intrauterine fetal demise.

\section{COPY NUMBER VARIATIONS}

CNVs are increasingly recognized as a major contributor to the pathogenesis of CHD. Estimates for the portion of CHD attributable to CNVs have been limited by the resolution of CNV detection methods and small study sizes. In a study of sporadic cases of complex CHD, high-density single nucleotide polymorphism (SNP) genotyping arrays and whole exome sequencing were used to identify CNVs with a limit of detection of $0.1 \mathrm{~KB}$ [11]. A de novo putative CNV was identified in approximately $10 \%$ of cases for whom a pathogenic genetic lesion was not already identified, including recurrently affected regions 1q21.1, 7q11.23, 8p23.1, 11q25, 15q11.2, and 22q11.2. In another study, hypoplastic left heart syndrome or conotruncal defect (CTD) patient/parent trios were prospectively recruited from a single institution without first screening for family history but incorporating echocardiographic data on all available parents [12]. Using array competitive genomic hybridization, a presumed causative CNV was identified in 5.6\% of probands. They, too, identified recurrent de novo CNVs affecting 1q21.1 and 22q11.2 regions, as well as 19p13.3 and many recurrent rare inherited CNVs. Interestingly, an association between rare inherited CNVs and underdiagnosed parental CHD was not observed, and, specifically for those parents found to have BAV, there was no association with their child having hypoplastic left heart syndrome. It was also worth noting that the two classes of CHD did not differ in their $\mathrm{CNV}$ incidence or the specific genes affected.

Rigorously discerning pathologic from incidental CNVs has been problematic. Higher resolution and larger-sized studies have improved our ability to implicate certain CNVs, but even well-designed studies have yielded conflicting results. In isolation, these results offer an unclear image of the biological impact of harboring any given CNV and, therefore, limit genotype-phenotype associations and predictions. To address this, $1694 \mathrm{CHD}$ cases collected from publicly available databases [Data-basE of Chromosomal Imbalance and 
Phenotype in Humans using Ensembl Resources (DECIPHER), The International Standards for Cytogenomic Arrays Consortium (ISCA), and CHDWiki], recent literature reviews, and institutional resources were reviewed to identify minimal critical domains and candidate genes based on known roles in heart development [13"']. In this study, GATA4 deletions were observed to have near complete CHD penetrance, whereas loss of other classic CHD genes (TBX1 and NOTCH1) had at most 50\% penetrance. In addition to the leading 22q11 deletion candidate gene, $T B X 1$, other implicated genes in this region include $C R K L$ and $M A P K 1$. Nonetheless, approximately $8 \%$ of CHD cases with a 22q11 microdeletion did not have loss of any of these three genes.

\section{GENE ENVIRONMENT INTERACTION}

An expanding list of environmental risk factors for CHD, including infectious, autoimmune, and toxic ones, have been proposed, most with only modest relative risk (RR) [14]. The phenotypic heterogeneity and incomplete penetrance characteristic of CHD genetics may remain esoteric unless modifying factors, such as environmental exposures, additional genetic variants, or epigenetic imprinting are evaluated. Folate supplementation during pregnancy, initiated to reduce risk of neural tube defects, unexpectedly reduced CHD incidence. Efforts to identify folate metabolic genetic variants associated with CHD risk have yielded inconsistent results. It is possible that this inconsistency may be a result of environmental confounders.

Fetal heart growth and remodeling is a time of rapid DNA replication. Toxins, such as those found in tobacco, are known to damage DNA and, logically, could impact heart development. Despite that, epidemiological studies on tobacco exposure and CHD incidence have not consistently observed a relationship or magnitude of influence $[15,16]$. Assuming tobacco exposure does increase CHD risk, a confounding variable might be obscuring the true associated risk. A recent study observed a small baseline risk of tobacco exposure for CHD [odds ratio (OR) 1.09, 95\% confidence interval (CI): 1.02-1.17] that was worsened by four different variants in the base excision repair gene OSGEP in the fetus (OR range 1.371.39 and each statistically significant) [17]. In contrast, protective maternal alleles were observed for the base excision repair gene $P A R P 2$ (OR=0.77, 95\% CI: 0.63-0.94) and the nucleotide excision repair genes $E R C C 1$ ( $\mathrm{OR}=0.76,95 \%$ CI: $0.62-0.94)$ and $E R C C 5$ $(\mathrm{OR}=0.61,95 \%$ CI: $0.42-0.87)$.

Tobacco smoke and maternal obesity were recently evaluated as environmental exposures interacting with both maternal and fetal variants of 60 genes involved in metabolic pathways associated with CTDs (folate, homocysteine, and trans-sulfuration) [18]. No SNP was associated with risk of CTD in the absence of tobacco exposure, and no protective SNP was identified. In mothers who smoked, SNPs in the glutathione-S-transferase (GST) family of genes, which mitigate oxidative stress, were found to increase risk of CTD, and the greatest impact was observed in GSTA4 (RR=1.74, 95\% CI: 1.21-2.49]. A SNP of GCLC, which encodes a rate-limiting enzyme in glutathione synthesis that contributes to DNA methylation and transsulfuration, in the fetus was also demonstrated to increase tobacco-associated CTD risk ( $\mathrm{RR}=2.12,95 \% \mathrm{CI}: 1.48-3.04)$. In two additional genes involved in DNA synthesis, a fetal and maternal SNP in RFC1 and a fetal SNP in NOS3 were associated with more 
modest increased CTD risk. Similar associations with CTD risk were observed in obese mothers with a maternal SNP in $G C L C$, different from the fetal SNP above (RR=2.00, 95\% CI: 1.41-2.83), and a fetal GSTA3 SNP (RR=1.83, 95\% CI: 1.33-2.52) roughly doubled the risk.

In contrast to tobacco exposure, the mechanisms whereby other nongenetic factors contribute to CHD pathogenesis, including maternal age and obesity, have been less clear. Using an Nkx2-5 mutant mouse known to exhibit ventricular septal defects (VSD), reciprocal ovarian transplantation was used to demonstrate that the association between maternal age and CHD may be linked to altered extrauterine environment (Fig. 2) [19"']. This effect was dependent on both genetic background and lifestyle factors. In contrast to wild type mice, hybrid strains of mice with protective genetic backgrounds and that differed on a number of metabolic traits were not found to have increasing VSD prevalence in their offspring with age. In aged mice, maternal exercise - particularly if performed at young age - lowered offspring VSD incidence without affecting glycemia, body mass, or adiposity. However, exercise did not alter VSD incidence in offspring of young mice. Irrespective of age, high-fat chow altered glycemia but did not impact VSD incidence. These data suggest a strong link between CHD incidence and extrauterine environment as determined by maternal age, genetic background, and exercise, but not actually with metabolic state. Although not consistently observed, both increasing maternal age and obesity have been previously associated with CHD in population-based studies, but maternal exercise is a relatively novel protective factor; additionally, ethnic background has been illustrated to impact the incidence of severe forms of CHD [20-22].

\section{CONGENITAL HEART DISEASE WITH EXTRACARDIAC DEFECTS}

The genetic mechanisms that determine whether a CHD presents in isolation (isoCHD) or with associated extracardiac defects (CHD+) remain largely unknown. For that matter, when a novel variant is identified in a patient with $\mathrm{CHD}+$, one must consider whether to implicate the lesion in CHD pathogenesis or to recognize it as being incidental. The most widely recognized associated defects for patients with CHD fall into the category of neurodevelopmental delays (NDD), and until recently this association was intuitively attributed to baseline oxygen delivery deficits during fetal and postnatal life or to secondary brain injury. A recent follow-up to the exome study of Zaidi and colleagues challenged this assertion.

WES was performed on 1220 nonsyndromic complex CHD trios recruited from Pediatric Cardiac Genetics Consortium or Pediatric Heart Network - dichotomized by presence of NDD or other congenital anomaly - and 900 control trios to identify de novo damaging missense and loss-of-function mutations [23"']. A statistically significant burden of functionally significant de novo mutations was observed in the subset of patients with extracardiac defects, particularly those who had NDD and one or more structural congenital anomalies, but not in isoCHD (19\% in CHD+NDD compared with 1.5\%). Filtering for mutations affecting genes highly expressed in the heart (HHE) during development increased this burden. Interestingly, filtering these variants instead for genes affected in one of six studies of diverse NDD or for genes highly expressed in the developing brain (HBE) also demonstrated increased burden. The most commonly affected biological processes 
involving genes that overlap the HBE and HHE lists included key gene expression processes such as chromatin modification, signal transduction, transcription regulation, and mRNA splice regulation.

The overlapping incidence of heart and cognitive development appears to result, at least in part, from the commonality of genes that regulate development of heart and brain. However, important questions remain to be answered. Although suggested by the observed genetic burden, it is challenging to conclude that isolated NDD, CHD with NDD, and CHD+ but not isoCHD can be attributed to de novo exonic mutations. After excluding clinically identified syndromic CHD, mutations affecting Noonan (PTPN11 and SOS1) and Kabuki (KMT2D) syndrome genes were still identified. With age, these children may eventually develop characteristic features of the respective syndromes. Pending further clinical follow-up, one must consider how to differentiate CHD+ from syndromic CHD with incomplete extracardiac penetrance when mutations affecting syndromic CHD genes are identified. For example, a nonsense mutation affecting PITX2, known to cause Axenfeld-Rieger syndrome (abnormal eye, teeth, and umbilical skin development, and CHD including atrial septal defects and atrioventricular valve anomalies), was recently reported as segregating with the presence of variable endocardial cushion defects and incomplete penetrance of AxenfeldRieger syndrome in a single kindred [21]. The factors that led to the observed pleiotropy in some but not all affected family members were not identified, and the CHD phenotypic heterogeneity was also not addressed.

In contrast to structural defects, NDD can be subtle and late presenting, offering opportunities for the actions of numerous environmental confounders. However, it should be noted that in the Pediatric Cardiac Genomics Consortium study discussed above, a genetic burden was observed in a subgroup analysis of the isoCHD cases too young (less than 12 months) to perform accurate developmental assessment [20]. This underscores the importance of increased suspicion for developmental delays and the potential utility of genetic testing even without a clearly perceived risk.

\section{CONCLUSION}

Answers beget more questions. The improving limits of detection and evolving interpretative methods for next generation sequencing and copy number analysis continue to identify novel genes and variants involved in CHD pathogenesis, informing our clinical use of these tools.

Although the list of associated genes and implicated variants continues to grow, the cause for most CHD cases remains challenging to identify and, for many implicated variants, the precise molecular mechanisms remain unexplored. Phenotypic heterogeneity and incomplete penetrance complicate our understanding of CHD genetics. However, it seems more likely than ever that our gaps in understanding the causes of CHD are primarily genetic and that the mechanisms are multifactorial.

\section{Acknowledgments}

Financial support and sponsorship

This work was partly funded by a grant from the National Heart Lung and Blood Institute (HL098123) to B.D.G. 


\section{REFERENCES AND RECOMMENDED READING}

Papers of particular interest, published within the annual period of review, have been highlighted as:

- of special interest

- of outstanding interest

1•. El Malti R, Liu H, Doray B, et al. A systematic variant screening in familial cases of congenital heart defects demonstrates the usefulness of molecular genetics in this field. Eur J Hum Genet. 2016; 24:228-236. Sequencing coding region GATA4, NKX2-5, ZIC3, and ELN and copy number analysis of 22q11 region, BMP4, CRELD1, NKX2-5, and TBX5 in familial CHD. [PubMed: 26014430]

2. Blue GM, Kirk EP, Giannoulatou E, et al. Targeted next-generation sequencing identifies pathogenic variants in familial congenital heart disease. J Am Coll Cardiol. 2014; 64:2498-2506. [PubMed: 25500235]

3-. Jia Y, Louw JJ, Breckpot J, et al. The diagnostic value of next generation sequencing in familial nonsyndromic congenital heart defects. Am J Med Genet A. 2015; 167A:1822-1829. Sequencing coding regions in 57 proven and candidate CHD genes in familial CHD. [PubMed: 25931334]

4. Weiss K, Applegate C, Wang T, Batista DA. Familial TAB2 microdeletion and congenital heart defects including unusual valve dysplasia and tetralogy of fallot. Am J Med Genet A. 2015; 167:2702-2706. [PubMed: 26139517]

5. Qu XK, Qiu XB, Yuan F, et al. A novel NKX2. 5 loss-of-function mutation associated with congenital bicuspid aortic valve. Am J Cardiol. 2014; 114:1891-1895. [PubMed: 25438918]

6. Bonachea EM, Zender G, White P, et al. Use of a targeted, combinatorial next-generation sequencing approach for the study of bicuspid aortic valve. BMC Med Genomics. 2014; 7:56. [PubMed: 25260786]

7-. Zaidi S, Choi M, Wakimoto $\mathrm{H}$, et al. De novo mutations in histone-modifying genes in congenital heart disease. Nature. 2013; 498:220-223. Whole exome sequencing of 362 sporadic CHD trios identified a likely causative, de-novo variant in approximately $10 \%$ of cases with an enrichment of epigenetic regulators. [PubMed: 23665959]

8. Arndt AK, MacRae CA. Genetic testing in cardiovascular diseases. Curr Opin Cardiol. 2014; 29:235-240. [PubMed: 24717670]

9--. Li Y, Klena NT, Gabriel GC, et al. Global genetic analysis in mice unveils central role for cilia in congenital heart disease. Nature. 2015; 521:520-524. Using a forward genetic screen and mouse embryonic imaging, recovered a spectrum of CHD mutant lines, including $30 \%$ with heterotaxy. [PubMed: 25807483]

10. Theis JL, Zimmermann MT, Evans JM, et al. Recessive MYH6 mutations in hypoplastic left heart with reduced ejection fraction. Circ Cardiovasc Genet. 2015; 8:564-571. [PubMed: 26085007]

11. Glessner JT, Bick AG, Ito K, et al. Increased frequency of de novo copy number variants in congenital heart disease by integrative analysis of single nucleotide polymorphism array and exome sequence data. Circ Res. 2014; 115:884-896. [PubMed: 25205790]

12. Warburton D, Ronemus M, Kline J, et al. The contribution of de novo and rare inherited copy number changes to congenital heart disease in an unselected sample of children with conotruncal defects or hypoplastic left heart disease. Hum Genet. 2014; 133:11-27. [PubMed: 23979609]

13-. Thorsson T, Russell WW, El-Kashlan N, et al. Chromosomal imbalances in patients with congenital cardiac defects: a meta-analysis reveals novel potential critical regions involved in heart development. Congenit Heart Dis. 2015; 10:193-208. Reviewed multiple CNV databases and institutional data to clarify inconsistencies between reports of CNV pathogenicity and identify critical gene or subregion. [PubMed: 24720490]

14. Jenkins KJ, Correa A, Feinstein JA, et al. Noninherited risk factors and congenital cardiovascular defects: current knowledge: a scientific statement from the American Heart Association Council on Cardiovascular Disease in the Young: endorsed by the American Academy of Pediatrics. Circulation. 2007; 115:2995-3014. [PubMed: 17519397] 
15. Alverson CJ, Strickland MJ, Gilboa SM, Correa A. Maternal smoking and congenital heart defects in the Baltimore-Washington Infant Study. Pediatrics. 2011; 127:e647-e653. [PubMed: 21357347]

16. Karatza AA, Giannakopoulos I, Dassios TG, et al. Periconceptional tobacco smoking and isolated congenital heart defects in the neonatal period. Int J Cardiol. 2011; 148:295-299. [PubMed: 19951824]

17. Tang X, Hobbs CA, Cleves MA, et al. Genetic variation affects congenital heart defect susceptibility in offspring exposed to maternal tobacco use. Birth Defects Res A Clin Mol Teratol. 2015; 103:834-842. [PubMed: 26033827]

18. Tang X, Nick TG, Cleves MA, et al. Maternal obesity and tobacco use modify the impact of genetic variants on the occurrence of conotruncal heart defects. PLoS One. 2014; 9:e108903. [PubMed: 25275547]

19-. Schulkey CE, Regmi SD, Magnan RA, et al. The maternal-age-associated risk of congenital heart disease is modifiable. Nature. 2015; 520:230-233. Employing a novel method of reciprocal ovarian transplantation in mice, investigates association of maternal age with CHD with extrauterine environment and maternal age in isolation. [PubMed: 25830876]

20. Blomberg MI, Kallen B. Maternal obesity and morbid obesity: the risk for birth defects in the offspring. Birth Defects Res A Clin Mol Teratol. 2010; 88:35-40. [PubMed: 19711433]

21. Pradat P, Francannet C, Harris JA, Robert E. The epidemiology of cardiovascular defects, Part I: a study based on data from three large registries of congenital malformations. Pediatr Cardiol. 2003; 24:195-221. [PubMed: 12632215]

22. Harris JA, Francannet C, Pradat P, Robert E. The epidemiology of cardiovascular defects, part 2: a study based on data from three large registries of congenital malformations. Pediatr Cardiol. 2003; 24:222-235. [PubMed: 12632214]

23-. Homsy J, Zaidi S, Shen Y, et al. De novo mutations in congenital heart disease with neurodevelopmental and other congenital anomalies. Science. 2015; 350:1262-1266. Identifies the genetic commonality between heart and brain development as a significant contributor to the clinical overlap between CHD and neurodevelopmental delays. [PubMed: 26785492] 


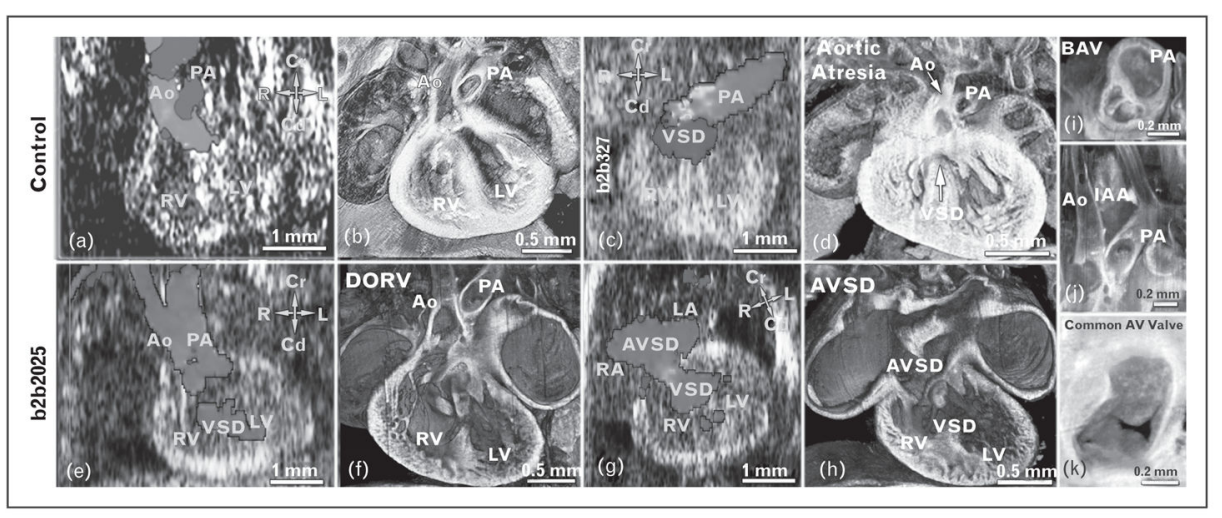

\section{FIGURE 1.}

Ultrasound diagnoses of CHD and cilia defects in CHD mutants. (a and b) Vevo 2100 color flow imaging showed crisscrossing of blood flow indicating normal aorta (Ao) and pulmonary artery alignment, confirmed by histopathology (b). Cd, caudal; Cr, cranial; L, left; LV, left ventricle; R, right; RV, right ventricle. (c and d) Embryonic day (e) 16.5 mutant mouse (line b2b327) exhibited a blood flow pattern indicating single great artery (pulmonary artery) and ventricular septal defect (VSD) (c), suggesting aortic atresia with ventricular septal defect, confirmed by histopathology (d). (e-h) Color flow imaging of E15.5 mutant mouse (line b2b2025) with heterotaxy (stomach on right) showed side by side aorta and pulmonary artery, with the aorta emerging from the right ventricle, indicating DORV/ ventricular septal defect (e and f) and the presence of AVSD ( $g$ and $h$ ). AVSD, atrioventricular septal defect; DORV, double outlet right ventricle; LA, left atrium; RA, right atrium. (i-k) Histopathology also showed a bicuspid aortic valve (BAV) (i), interrupted aortic arch (IAA) (j), and common atrioventricular valve (k). Reproduced with permission from [9"']. 


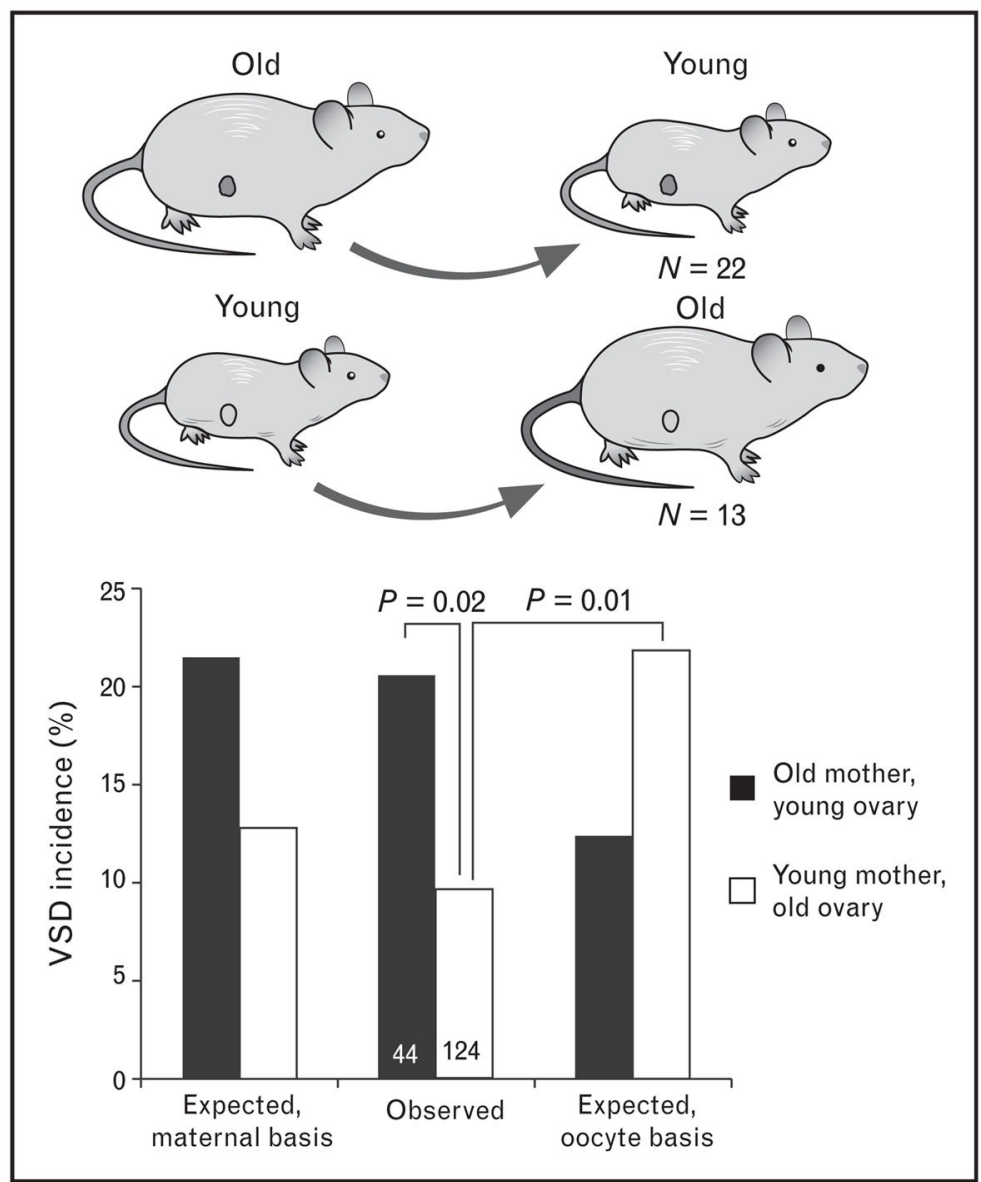

FIGURE 2.

Reciprocal ovarian transplants between young and old mothers localize the basis of the maternal-age-associated risk to the mother. The incidence of ventricular septal defects for the offspring of old mothers with young ovaries is significantly greater than that of young mothers with old ovaries. The observed incidence in the offspring of recipient mothers matches that expected for a maternal but not an oocyte basis of the age effect. The observed and expected incidences were compared in a chi-squared goodness-of-fit test. The number of recipient mothers and the number of pups in each age group are shown. Reproduced with permission from [19"']. VSD, ventricular septal defect. 